\title{
On the Automorphisms of the Four-dimensional Real Division Algebras
}

\author{
André S. Diabang ${ }^{1}$, Alassane Diouf ${ }^{1}$, Mankagna A. Diompy ${ }^{1} \&$ Alhousseynou Ba $^{1}$ \\ ${ }^{1}$ Département de Mathématiques et Informatiques, Faculté des Sciences et Techniques, Université Cheikh Anta Diop, \\ Dakar, Sénégal \\ Correspondence: André S. Diabang, Département de Mathématiques et Informatiques, Faculté des Sciences et Tech- \\ niques, Université Cheikh Anta Diop, Dakar, Sénégal. E-mail: andrediabang@yahoo.fr
}

Received: December 8, 2016 Accepted: January 18, 2017 Online Published: March 21, 2017

doi:10.5539/jmr.v9n2p95

URL: https://doi.org/10.5539/jmr.v9n2p95

\begin{abstract}
In this paper, we study partially the automorphisms groups of four-dimensional division algebra. We have proved that there is an equivalence between $\operatorname{Der}(A)=s u(2)$ and $\operatorname{Aut}(A)=S O(3)$. For an unitary four-dimensional real division algebra, there is an equivalence between $\operatorname{dim}(\operatorname{Der}(A))=1$ and $\operatorname{Aut}(A)=S O(2)$.
\end{abstract}

Keywords: division algebra, derivations, automorphisms, mutation, isotope.

\section{Introduction}

The finited-dimensional real division algebra A, an actuel problem, takes its origin with the quaternion's discovery $\mathbb{H}$, by Hamilton in 1843. One of the fundementals results of a n-dimensional real division algebra affirms that $n \in\{1,2,4,8\}$ (Bott $\&$ Milnor, 1958; Kervaire, 1958). For $n \in\{1,2\}$, the real division algebra $A$ is known (Althoen \& Kugler, 1983; Hübner \& Peterson, 2004; Dieterich, 2005). However the problem persists for the others cases. One of the method of determining the algebra $\mathrm{A}$ is to know its derivations and/or its automorphisms. Benkart and Osborn have classified Lie algebra of derivations $\operatorname{Der}(A)$ (Benkart \& Osborn, 1981). It's well known that if $A$ is finite dimensional ,then the automorphism group $\operatorname{Aut}(A)$ is a group of Lie, whose associated Lie algebra and Lie algebra $\operatorname{Der}(A)$ coincide. In dimension 1 , the group $\operatorname{Aut}(A)$ is trivial. In dimension 2, Dieterich has classified $A u t(A)$, (Dieterich, 2005). However the problem persists for the others cases. This paper is a contribution to the advancement of the determination of the group $\operatorname{Aut}(A)$. In the first part, we give some preliminaries results on the automorphism of an algebra A. In the second part, we characterize the 4-dimensional real division algebra $A$ whose $A u t(A)=S O(3)$. Finally, we characterize also an unitary 4-dimensional real division algebra whose $A u t(A)=S O(2)$.

\section{Preliminary}

An algebra is said to be mutation $\alpha$ of $A$ denoted $A^{\alpha}$, the vector space A which has as product: $x \bullet \alpha=\alpha x y+(1-\alpha) y x, x$, $y \in A$. If $\lambda, \mu \in \mathbb{R}$ we have $\left(A^{\lambda}\right)^{\mu}=A^{\alpha}$ with $\alpha=2 \lambda \mu-\lambda-\mu+1$. The product of $\mathbb{H}^{\lambda}$ in the basic $e=1, e_{1}=\frac{i}{2 \lambda-1}, e_{2}=\frac{j}{2 \lambda-1}$, $e_{3}=\frac{k}{2 \lambda-1}$, is given by: $e e_{n}=e_{n} e=e_{n} ; e_{n}^{2}=\frac{1}{(2 \lambda-1)^{2}} e ; e_{1} e_{2}=-e_{2} e_{1}=e_{3} ; e_{1} e_{3}=-e_{3} e_{1}=-e_{2} ; e_{2} e_{3}=-e_{3} e_{2}=e_{1}$. Where $\{1, i, j, k\}$ in the canonical basis of the quaternions algebra $\mathbb{H}$. We denote $\operatorname{Aut}(A)=\{f: A \longrightarrow A$, linear bijection: $f(x y)=f(x) f(y), \forall x y \in A\}$ the automorphism group of $A$. We denote $\operatorname{Der}(A)=\{\partial: A \longrightarrow A$, linear mapping: $\partial(x y)=\partial(x) y+x \partial(y), \forall x y \in A\}$ the Lie algebra of derivations of $A$. The algebra $A$ is called division if for all $x \in A-\{0\}$ the linears mapping $L_{x}$ and $R_{x}$ are bijective. Let $x, y \in A,[x, y]=x y-y x$ is the commutator of $x$ and $y$. We recall that $I(A)=\left\{x \in A: x^{2}=x\right\}$. Let $\phi, \psi$ the linears bijections, we call isotopy of $A$ denoted $A_{\phi, \psi}$, the algebra whose product is: $x \odot y=\phi(x) \psi(y), x, y \in A$.

Example The mutation $\lambda \in \mathbb{R}$ of $\mathbb{C}, \mathbb{C}^{\lambda}$ is isomorphic to $\mathbb{C}$. The mutation $\frac{1}{2}$ of $\mathbb{H}$, $\mathbb{H}^{\frac{1}{2}}$ is commutative and it's not of division, called the symtrization, one notes it $\mathbb{H}^{+}$

Lemma 1 Let $A$ be a real algebra, then the following assertions are equivalent:

1. $f \in \operatorname{Aut}(A)$ and $[f, \varphi]=[f, \psi]=0$;

2. $f \in \operatorname{Aut}\left(A_{\phi, \psi}\right)$ and $[f, \varphi]=[f, \psi]=0$. 
Proof. Let $f \in \operatorname{Aut}\left(A_{\phi, \psi}\right)$, for all $x$ and $y \in A$ we have:

$$
\begin{aligned}
f(x \odot y) & =f(x) \odot f(y) \\
\Leftrightarrow f(\phi(x) \cdot \psi(y)) & =\varphi(f(x)) \cdot \psi(f(y)) \\
\Leftrightarrow f(\phi(x) \cdot \psi(y)) & =f(\phi(x)) \cdot f(\psi(y)) . \text { Then } f \in \text { Aut }(A) .
\end{aligned}
$$

Lemma 2 Let $A$ be an algebra and $\lambda \in \mathbb{R}$, so Aut $(A) \subset \operatorname{Aut}\left(A^{(\lambda)}\right)$. Furthermore if $\lambda \neq \frac{1}{2}$ then Aut $(A)=A u t\left(A^{(\lambda)}\right)$.

Proof. It's easy to show that $\operatorname{Aut}(A) \subset \operatorname{Aut}\left(A^{(\lambda)}\right)$. If $\lambda \neq \frac{1}{2}$, we have $\operatorname{Aut}\left(A^{(\lambda)}\right) \subset \operatorname{Aut}\left(\left(A^{(\lambda)}\right)^{\frac{\lambda}{2 \lambda-1}}\right)=\operatorname{Aut}(A)$

\section{Characterization of Four-dimensional Real Division Algebra with $S O(3)$ as Its Automorphic Group}

In (Benkart \& Osborn, $\left.(1981)_{2}\right)$, we have the following result:

Theorem $1 A$ is an four-dimensional real division algebra with su(2) as its derivation algebra if and only if $A$ has a basis $\left\{e, e_{1}, e_{2}, e_{3}\right\}$ with multiplication given by (1.1) for some real numbers $\alpha, \beta$, $\gamma$ such that $\alpha \beta \gamma>0$.

$$
\begin{array}{r}
e^{2}=e, \quad e e_{i}=\alpha e_{i}, \quad e_{i} e=\beta e_{i} \quad e_{i}^{2}=-\gamma e \text { for all } i \in\{1,2,3\} \\
e_{1} e_{2}=-e_{2} e_{1}=e_{3}, \quad e_{2} e_{3}=-e_{3} e_{2}=e_{1}, \quad e_{3} e_{1}=-e_{1} e_{3}=e_{2}
\end{array} .
$$

Remark 1 Let $x=\lambda_{0} e+\lambda_{1} e_{1}+\lambda_{2} e_{2}+\lambda_{3} e_{3}, y=\lambda_{0}^{\prime} e+\lambda_{1}^{\prime} e_{1}+\lambda_{2}^{\prime} e_{2}+\lambda_{3}^{\prime} e_{3} \in A$, we have:

$$
\begin{aligned}
x y & =\left(\lambda_{0} \lambda_{0}^{\prime}-\gamma \lambda_{1} \lambda_{1}^{\prime}-\gamma \lambda_{2} \lambda_{2}^{\prime}-\gamma \lambda_{3} \lambda_{3}^{\prime}\right) e+\left(\alpha \lambda_{0} \lambda_{1}^{\prime}+\beta \lambda_{1} \lambda_{0}^{\prime}+\lambda_{2} \lambda_{3}^{\prime}-\lambda_{3} \lambda_{2}^{\prime}\right) e_{1} \\
& +\left(\alpha \lambda_{0} \lambda_{2}^{\prime}+\beta \lambda_{2} \lambda_{0}^{\prime}+\lambda_{3} \lambda_{1}^{\prime}-\lambda_{1} \lambda_{3}^{\prime}\right) e_{2}+\left(\alpha \lambda_{0} \lambda_{3}^{\prime}+\beta \lambda_{3} \lambda_{0}^{\prime}+\lambda_{1} \lambda_{2}^{\prime}-\lambda_{2} \lambda_{1}^{\prime} .\right) e_{3}
\end{aligned}
$$

We defined $\psi_{\alpha}: A \longrightarrow A ; \psi_{\alpha}(\lambda e+u)=\lambda e+\frac{1}{\alpha} u$ with $(\alpha, \lambda) \in \mathbb{R}^{*} \times \mathbb{R}$ and $u \in \operatorname{lin}\left\{e_{1}, e_{2}, e_{3}\right\}$.

Theorem 2 Let $A$ be an 4-dimensional real division algebra with su(2) as its derivation algebra, then the isotope $A_{\psi_{\alpha}, \psi_{\beta}}$ of $A$ is isomorphic to $\mathbb{H}^{\mu}$ with $\mu=\frac{1}{2 \sqrt{\alpha \beta \gamma}}+\frac{1}{2}$.

Proof. Let $A$ be an algebra of theorem 1. The multiplication of $A_{\psi_{\beta}, \psi_{\alpha}}$ in the basis $\left\{e, e_{1}, e_{2}, e_{3}\right\}$ is given by (1.2)

$$
\begin{gathered}
e \odot e=e, \quad e \odot e_{i}=e_{i} \odot e=e_{i}, \quad e_{i} \odot e_{i}=-\frac{\gamma}{\alpha \beta} e \text { for all } i \in\{1,2,3\} \\
e_{1} \odot e_{2}=-e_{2} \odot e_{1}=\frac{1}{\alpha \beta} e_{3}, \quad e_{2} \odot e_{3}=-e_{3} \odot e_{2}=\frac{1}{\alpha \beta} e_{1}, \quad e_{3} \odot e_{1}=-e_{1} \odot e_{3}=\frac{1}{\alpha \beta} e_{2}
\end{gathered}
$$

Setting $e^{\prime}=e, e_{1}^{\prime}=\alpha \beta e_{1}, e_{2}^{\prime}=\alpha \beta e_{2}$ and $e_{3}^{\prime}=\alpha \beta e_{3}$, we obtain, an algebra isomorphic to $\mathbb{H}^{\mu}$ with $\mu=\frac{1}{2 \sqrt{\alpha \beta \gamma}}+\frac{1}{2}$.

Corollary 1 Every four-dimensional real division algebra with su(2) as its derivation algebra is isotope to the algebra $\mathbb{H}^{\lambda}$.

Lemma 3 Let $A$ be an 4-dimensional real division algebra with su(2) as its derivation algebra. Then A has a basis $\left\{e, e_{1}, e_{2}, e_{3}\right\}$ with multiplication given by (1.1). Then we have

$$
\begin{aligned}
& I(A)=\{e\} \cup\left\{\frac{1}{\alpha+\beta} e+\sum_{i=1}^{3} \lambda_{i} e_{i} ; \sum_{i=1}^{3} \lambda_{i}^{2}=\frac{1-(\alpha+\beta)}{\gamma(\alpha+\beta)^{2}}\right\}, \text { if } \alpha+\beta \neq 0 \text { and } \frac{1-(\alpha+\beta)}{\gamma}>0, \\
& I(A)=\{e\}, \text { otherwise. }
\end{aligned}
$$

Proof. Let $x=\lambda_{0} e+\lambda_{1} e_{1}+\lambda_{2} e_{2}+\lambda_{3} e_{3} \in A$, we have:

$$
x^{2}=x \Longleftrightarrow\left\{\begin{array}{l}
\lambda_{0}^{2}-\gamma\left(\lambda_{1}^{2}+\lambda_{2}^{2}+\lambda_{3}^{2}\right)=\lambda_{0} \\
\lambda_{i}\left((\alpha+\beta) \lambda_{0}-1\right)=0, \quad i \in\{1,2,3\}
\end{array}\right.
$$

We obtain $I(A)$ by resolving the system and discussing on $\alpha+\beta$ and $\frac{1-(\alpha+\beta)}{\gamma}$. 
Corollary 2 Let $A$ be an real algebra of theorem 1. Let $u$ and $v \in$ A linearly independent. Then the following assertions are equivalent:

1. $x \in I(A), u^{2}=v^{2}=-\gamma x, x u=\alpha u, u x=\beta u, x v=\alpha v$, and $v x=\beta v$

2. $x=e$ and $u, v \in\left\{\lambda_{1} e_{1}+\lambda_{2} e_{2}+\lambda_{3} e_{3} ;\right.$ with $\left.\lambda_{1}^{2}+\lambda_{2}^{2}+\lambda_{3}^{2}=1\right\}$.

Proof. (1) $\Longrightarrow(2)$ the proof will be reduce in the case $\alpha+\beta \neq 0$ and $\frac{1-(\alpha+\beta)}{\gamma}>0$.

Suppose that $x=\frac{1}{\alpha+\beta} e+\lambda_{1} e_{1}+\lambda_{2} e_{2}+\lambda_{3} e_{3} \in I(A)$ with $\sum_{i=1}^{3} \lambda_{i}^{2}=\frac{1-(\alpha+\beta)}{\gamma(\alpha+\beta)^{2}}$.

Let $u=\Sigma_{i=0}^{3} \lambda_{i}^{\prime} e_{i}$, and $v=\Sigma_{i=0}^{3} \lambda_{i}^{\prime \prime} e_{i} \in A$ satisfied the equations of (a). We have:

$u^{2}=v^{2}=-\gamma x \Longrightarrow \lambda_{i}=-\frac{\alpha+\beta}{\gamma} \lambda^{\prime}{ }_{0} \lambda^{\prime}{ }_{i}=-\frac{\alpha+\beta}{\gamma} \lambda^{\prime \prime}{ }_{0} \lambda^{\prime \prime}{ }_{i} \quad i \in\{1,2,3\}$.

And $x u=\alpha u, x v=\alpha v \Longrightarrow \lambda_{0}^{\prime 2}=\lambda_{0}^{\prime \prime 2}=\frac{\alpha \gamma(1-(\alpha+\beta))}{\beta(\alpha+\beta)^{2}}$. Consequently $\lambda_{0}^{\prime}=\varepsilon \lambda_{0}^{\prime \prime}$ with $\varepsilon^{2}=1$. We have $u=\varepsilon v$ according to (E.1), which is adsurd since $u$ and $v$ are linearly independent, then $x=e$. It's easily shown that the equations $u^{2}=v^{2}=-\gamma e, e u=\alpha u, u e=\beta u, e v=\alpha v$, and $v e=\beta v$ gives $\lambda_{0}^{\prime}=\lambda_{0}^{\prime \prime}=0$ and $\Sigma_{i=0}^{3} \lambda_{i}^{\prime 2}=\Sigma_{i=0}^{3} \lambda_{i}^{\prime \prime 2}=1$.

(2) $\Longrightarrow(1)$ the proof is evident.

Proposition 1 Let $A$ be a 4-dimensional real division algebra with su(2) as its derivation algebra and $f \in$ Aut(A), then $f(e)=e$ and $f\left(\operatorname{lin}\left\{e_{1}, e_{2}, e_{3}\right\}\right) \subseteq \operatorname{lin}\left\{e_{1}, e_{2}, e_{3}\right\}$. Moreover $\left[f, \psi_{\alpha}\right]=0$.

Proof. We notice that $f(e) \in I(A)$ and $f\left(e_{i}\right)$ for all $i \in\{1,2,3\}$, satisfy to (a) of corollary 1 . Then $f(e)=e$ and $f\left(e_{i}\right) \in \operatorname{lin}\left\{e_{1}, e_{2}, e_{3}\right\}$. It's easy to show that $\left[f, \psi_{\alpha}\right]=0$.

Theorem 3 Let A be a 4-dimensional real division algebra with su(2) as its derivation algebra, then the following propositions are equivalent:

1. $\operatorname{Aut}(A) \cong S O(3)$;

2. $\operatorname{Der}(A) \cong \operatorname{su}(2)$;

3. $A_{\psi_{\alpha}, \psi_{\beta}}$ is isomorphic to $\mathbb{H}^{\mu}$ with $\mu=\frac{1}{2 \sqrt{\alpha \beta \gamma}}+\frac{1}{2}$.

Proof. $(1) \Longrightarrow(2) \operatorname{Der}(A)=\operatorname{Lie}(\operatorname{Aut}(A))=\operatorname{Lie}(S O(3)) \cong \operatorname{so}(3) \cong \operatorname{su}(2)$.

(2) $\Longrightarrow$ (3) See the Theorem 2 .

(3) $\Longrightarrow$ (1) All automorphisms of $A$ commute with $\psi_{\alpha}$ and $\psi_{\beta}$ according to Proposition 1 and also all automorphisms of $A_{\psi_{\alpha}, \psi_{\beta}}$ commute with $\psi_{\alpha}$ and $\psi_{\beta}$ according to theorem 2 , then $\operatorname{Aut}(A)=\operatorname{Aut}\left(A_{\psi_{\alpha}, \psi_{\beta}}\right)$.

The Lemmas 1 and 2 give $\operatorname{Aut}(A)=\operatorname{Aut}\left(A_{\psi_{\beta}, \psi_{\alpha}}\right)=\operatorname{Aut}\left(\mathbb{H}^{\mu}\right)=\operatorname{Aut}(\mathbb{H}) \cong S O(3)$.

\section{Characterization Unitary 4-dimensional Real Division Algebra with $S O(2)$ as Its Automorphisms Groups}

In (Diabang \& all, (2016) 1 ), we have the following result:

Theorem 4 Let $A$ be an unital 4-dimensional real division algebra having a non-trivial derivation $\partial$, then there exists a basis $\mathcal{B}_{1}=\left\{e, e_{1}, e_{2}, e_{3}\right\}$ of $A$ for which the multiplication is given by the table (1.3):

\begin{tabular}{|c|c|c|c|c|}
\hline$\odot$ & $e$ & $e_{1}$ & $e_{2}$ & $e_{3}$ \\
\hline$e$ & $e$ & $e_{1}$ & $e_{2}$ & $e_{3}$ \\
\hline$e$ & $e_{1}$ & $-e$ & $\alpha_{1} e_{2}+\alpha_{2} e_{3}$ & $-\alpha_{2} e_{2}+\alpha_{1} e_{3}$ \\
\hline$e$ & $e_{2}$ & $\alpha_{3} e_{2}+\alpha_{4} e_{3}$ & $\alpha_{5} e+\alpha_{6} e_{1}$ & $\alpha_{7} e+\alpha_{8} e_{1}$ \\
\hline 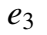 & $e_{3}$ & $-\alpha_{4} e_{2}+\alpha_{3} e_{3}$ & $-\alpha_{7} e-\alpha_{8} e_{1}$ & $\alpha_{5} e+\alpha_{6} e_{1}$ \\
\hline
\end{tabular}

for some real numbers $\alpha_{i}, i \in\{1, \ldots, 7\}$.

Corollary 3 Let A be an four-dimensional real unital division algebra A having a non-trivial derivation, then the following propositions are equivalent:

1. $\alpha_{1}=\alpha_{3}=\alpha_{6}=\alpha_{7}=0, \alpha_{5}<0, \alpha_{2}=-\alpha_{4} \neq 0$ and $\alpha_{8}=-\alpha_{2} \alpha_{5} \neq 0$;

2. A is quadratic and flexible;

3. $\operatorname{Der}(A)=\operatorname{su}(2)$; 
4. $\operatorname{Aut}(A)=S O(3)$;

5. A is isotope to $\mathbb{H}^{\mu}$.

Proof. (1) $\Longleftrightarrow(2) \Longleftrightarrow$ (3) results of Theorem 2 in (Diabang \& all, (2016) 1 ).

(3) $\Longleftrightarrow(4) \Longleftrightarrow(5)$ results of Theorem 3 .

Lemma 4 Let $A$ be an unital four-dimensional real division algebra having a non-trivial derivation $\partial$ such that $A$ isn't quadratic or isn't flexible. If $f \in \operatorname{Aut}(A)$, then $f(e)=e$ and $f\left(e_{1}\right)=\varepsilon e_{1}$ with $\varepsilon^{2}=1$.

Proof. $f$ being bijective then for all $y \in A$ there is $x \in A$ such that $f(x)=y$. We have $f(e) y=f(e) f(x)=f(e x)=f(x)=y$ and $y f(e)=f(x) f(e)=f(x e)=f(x)=y$, then $f(e)$ is an unitary element of $A$, therefore $f(e)=e$. The subalgebra of $A$ generated by $f\left(e_{1}\right)$, denoted $\left\langle f\left(e_{1}\right)>\right.$, is isomorphic to $B_{0}=\operatorname{ker} \partial$. As $\operatorname{dim}(\operatorname{Der}(A))=1$ then for all $x \in<f\left(e_{1}\right)>$, $\partial(x)=0$ consequently $f\left(e_{1}\right) \in B_{0}$. The equation $f\left(e_{1}\right)^{2}=-e$ gives $f\left(e_{1}\right)=\varepsilon e_{1}$.

Remark 2 Let A be an unital 4-dimensional real division algebra having a non-trivial derivation. Let $x=\lambda_{0} e+\lambda_{1} e_{1}+$ $\lambda_{2} e_{2}+\lambda_{3} e_{3} \in A$, we have:

$$
\begin{aligned}
& x^{2}=-e \Longleftrightarrow\left\{\begin{array}{l}
\lambda_{0}^{2}-\lambda_{1}^{2}+\alpha_{5}\left(\lambda_{2}^{2}+\lambda_{3}^{2}\right)=-1 \\
2 \lambda_{0} \lambda_{1}+\alpha_{6}\left(\lambda_{2}^{2}+\lambda_{3}^{2}\right)=0 \\
2 \lambda_{0} \lambda_{2}+\left(\alpha_{1}+\alpha_{3}\right) \lambda_{1} \lambda_{2}-\left(\alpha_{2}+\alpha_{4}\right) \lambda_{1} \lambda_{3}=0, \\
2 \lambda_{0} \lambda_{3}+\left(\alpha_{2}+\alpha_{4}\right) \lambda_{1} \lambda_{2}+\left(\alpha_{1}+\alpha_{3}\right) \lambda_{1} \lambda_{3}=0 .
\end{array}\right. \\
& \lambda_{2} \mathbf{E} .4+\lambda_{3} \mathbf{E} .5 \\
& \lambda_{3} \mathbf{E} . \mathbf{4}+\lambda_{2} \mathbf{E} . \mathbf{5}=\left(2 \lambda_{0}+\left(\alpha_{1}+\alpha_{3}\right) \lambda_{1}\right)\left(\lambda_{2}^{2}+\lambda_{3}^{2}\right)=0
\end{aligned}
$$

There are four possible cases:

Cas 1. If $\alpha_{6}\left(\alpha_{2}+\alpha_{4}\right) \neq 0$, then $x^{2}=-e \Longleftrightarrow x=\varepsilon e_{1}$.

Cas 2. If $\alpha_{6}=0$ and $\alpha_{2}+\alpha_{4} \neq 0$, then

$$
x^{2}=-e \Longleftrightarrow\left\{\begin{array}{l}
x \in\left\{\varepsilon e_{1}\right\} \cup\left\{\lambda_{2} e_{2}+\lambda_{3} e_{3} ; \quad \lambda_{2}^{2}+\lambda_{3}^{2}=-\frac{1}{\alpha_{5}}\right\}, \quad \text { If } \quad \alpha_{5}<0 \\
x=\varepsilon e_{1} \quad \text { otherwise, }
\end{array}\right.
$$

Cas 3. If $\alpha_{6}=\alpha_{2}+\alpha_{4}=0$, then

$$
x^{2}=-e \Longleftrightarrow\left\{\begin{array}{l}
x \in\left\{\lambda_{1} e_{1}+\lambda_{2} e_{2}+\lambda_{3} e_{3} ; \quad \lambda_{1}^{2}=1+\alpha_{5} \lambda_{2}^{2}+\alpha_{5} \lambda_{3}^{2}\right\}, \quad \text { If } \alpha_{1}+\alpha_{3}=0 \\
x \in\left\{\varepsilon e_{1}\right\} \cup\left\{\lambda_{2} e_{2}+\lambda_{3} e_{3} ; \lambda_{2}^{2}+\lambda_{3}^{2}=-\frac{1}{\alpha_{5}}\right\}, \quad \text { If } \alpha_{1}+\alpha_{3} \neq 0 \text { and } \alpha_{5}<0 \\
x=\varepsilon e_{1}, \quad \text { If } \alpha_{1}+\alpha_{3} \neq 0 \text { and } \alpha_{5} \geq 0
\end{array}\right.
$$

Cas 4. If $\alpha_{6} \neq 0$ and $\alpha_{2}+\alpha_{4}=0$, then

$$
x^{2}=-e \Longleftrightarrow\left\{\begin{array}{l}
x \in\left\{\varepsilon e_{1}\right\} \cup\left\{k_{o} e+\varepsilon \sqrt{k_{1}} e_{1}+\lambda_{2} e_{2}+\lambda_{3} e_{3} ; \lambda_{2}^{2}+\lambda_{3}^{2}=\frac{\alpha_{1}+\alpha_{3}}{\alpha_{6}} k_{1}\right\}, \text { If } \frac{\alpha_{1}+\alpha_{3}}{\alpha_{6}}>0, k_{1}>0 \\
x=\varepsilon e_{1}, \text { otherwise }
\end{array}\right.
$$

with $k_{1}=\frac{4 \alpha_{6}}{4 \alpha_{6}-4 \alpha_{5}\left(\alpha_{1}+\alpha_{3}\right)-\alpha_{6}\left(\alpha_{1}+\alpha_{3}\right)^{2}}, k_{0}=-\frac{\varepsilon\left(\alpha_{1}+\alpha_{3}\right) \sqrt{k_{1}}}{2}$ and $\varepsilon \in\{-1,1\}$.

Proposition 2 Let $A$ be an unital four-dimensional real division algebra having a non-trivial derivation $\partial$ such that A isn't quadratic or isn't flexible. If $f \in \operatorname{Aut}(A)$, then

$$
M\left(f, \mathcal{B}_{1}\right)=\left(\begin{array}{cccc}
1 & 0 & 0 & 0 \\
0 & 1 & 0 & 0 \\
0 & 0 & \cos (\theta) & -\sin (\theta) \\
0 & 0 & \sin (\theta) & \cos (\theta)
\end{array}\right) .
$$

with $\theta \in \mathbb{R}$ so $A u t(A) \cong S O(2)$.

Proof. The lemma 4, gives $f(e)=e$ and $f\left(e_{1}\right)=\varepsilon e_{1}$. By the definition of the automorphism $f$ and the equations $(E .2), \ldots(E .7)$, we obtains the result. 
Definition 1 (Unit-duplication process) Let $B$ be an real algebra having an unit element e and let $\rho, \sigma, \phi, \psi: B \longrightarrow B$ be linear mappings such that $\phi(e)=\psi(e)=e$. We define on the space $B \times B$ the produit:

$$
(x, y) \odot\left(x^{\prime}, y^{\prime}\right)=\left(x x^{\prime}+\rho\left(\sigma\left(y^{\prime}\right) y\right) ; y \phi\left(x^{\prime}\right)+y^{\prime} \psi(x)\right)
$$

The algebra resulting has an unit element $(e, 0)$ and contains $B \times\{0\}$ as sub-algebra. It is said to be obtained from $B$ and $\varrho$, by unit-duplication process and is denoted by $U_{D P}(\rho, \sigma, \phi, \psi)$. This generalizes the classical Cayley-Dickson process as-well as the process given.

Theorem 5 Let $A$ be an unital 4-dimensional real division algebra having a non-trivial derivation such that A isn't quadratic or isn't flexible, then the following propositions are equivalent:

1. $\operatorname{Aut}(A) \cong S O(2)$;

2. $\operatorname{dim}(\operatorname{Der}(A))=1$;

3. A is obtained from the unital real algebra $\mathbb{C}$ by unit-duplication process.

Proof. $(1) \Longrightarrow(2) \operatorname{Der}(A)=\operatorname{Lie}(\operatorname{Aut}(A))=\operatorname{Lie}(S O(2))=\operatorname{so}(2), \operatorname{so} \operatorname{dim}(\operatorname{Der}(A))=1$.

(2) $\Longrightarrow$ (3) See Corollary 1 in (Diabang \& all, (2016) $)_{1}$.

(3) $\Longrightarrow$ (1) $A$ admits a nonzero derivation, then $A$ satisfies the hypotheses of the Theoreml 4 . The proposition 2 completes the proof.

Remark 3 Let A be a finite-dimensional real division algebra, whose Lie algebra of derivations is trivial, then the group $\operatorname{Aut}(A)$ is finite.

Problem 1 Let A be an four-dimensional real division algebra, whose group Aut $(A)$ is finite. Is there an upper limit to the order of the group Aut(A)?.

\section{References}

Althoen, S. C., \& Kugler, L. D. (1983). When is $\mathbb{R}^{2}$ a division algebra? Amer. Math. Monthly, 90, 625-635. https://doi.org/10.2307/2323281

Benkart, G. M., \& Osborn, J. M. (1981)1. The derivation algebra of a real division algebra. Amer. J. Math., 103, 11351150. https://doi.org/10.2307/2374227

Benkart, G. M., \& Osborn, J. M. (1981)2. An investigation of real division algebras using derivations. Pacific J. Math., 96, 265-300. https://doi.org/10.2140/pjm.1981.96.265

Bott, R., \& Milnor, J. (1958). On the parallelizability of the spheres. Bull. Amer. Math. Soc., 64, 87-89.

Diabang, A. S., Diankha, O., Ly, M., \& Rochdi, A. (2016) $)_{1}$. A note on the real division algebras with non-trivial derivations. International Journal of Algebra, 1(11).

Diabang, A. S., Diankha, O., \& Rochdi, A. (2016)2. On the automorphisms of absolute-valued algebras. International Journal of Algebra, 113 - 123.

Dieterich, E. (2005). Classification, automorphism groups and categorical structure of the two-dimensional real division algebras. Journal of Algebra and its Applications, 4, 517-538. https://doi.org/10.1142/S0219498805001307

Doković, D. Z̆., \& Zhao, K. (2004). Real division algebras with large automorphism group. J. Algebra., 282, 758-796

Jacobson, N. (1958). Composition algebras and their automorphisms. Rend. Cire. Mat. Palermo, 7(2), 55-80.

Hbner, M., \& Peterson, H. P. (2004). Two-dimensional real division algebras revisited. Beitrge Algebra Geom., 45, $29-36$.

M. Kervaire, (1958). Non-parallelizability of the $n$-sphere for $n>7$. Proc. Nat. Acad. Sci. USA, 44, $280-283$.

Rochdi, A. (1995). Etude des algbres relles de Jordan non commutatives, de division, de dimension 8, dont l'algbre de Lie des drivations n'est pas triviale. Journal of Algebra, 178, 843-871. https://doi.org/10.1006/jabr.1995.1381

\section{Copyrights}

Copyright for this article is retained by the author(s), with first publication rights granted to the journal.

This is an open-access article distributed under the terms and conditions of the Creative Commons Attribution license (http://creativecommons.org/licenses/by/4.0/). 